\title{
A KOROVKIN TYPE THEOREM ON WEAK CONVERGENCE
}

\author{
A. MEIR
}

ABSTRACT. A weak convergence theorem is proved for a sequence of positive contractions on $L_{1}(0,1)$.

In this note we shall prove the following result.

THEOREM. Let $\left\{T_{n}\right\}_{n=1}^{\infty}$ be a sequence of positive and contractive linear operators on $L_{1}(0,1)$, satisfying

$$
T_{n} 1 \stackrel{w}{\longrightarrow} 1, \quad T_{n} x \stackrel{w}{\longrightarrow} x
$$

Then

$$
T_{n} f \stackrel{w}{\longrightarrow} f
$$

for every $f \in L_{1}(0,1)$.

For the proof we shall need a classical result.

Lemma [1, Corollary IV. 8.11]. A subset $S$ of $L_{1}(0,1)$ is weakly sequentially compact if and only if it is bounded and

$$
\lim _{\mu E \rightarrow 0} \int_{E} f=0
$$

uniformly for $f \in S$.

Proof of the Theorem. Let $r, 0 \leqslant r \leqslant 1$, be fixed. Since $|x-r| \leqslant 1$ on $(0,1)$, we have by the positivity of the operators for any measurable $E \subset(0,1)$

$$
\int_{E} T_{n}(|x-r|) \leqslant \int_{E} T_{n} 1
$$

By the Lemma, the right-hand integral tends to zero, uniformly for $n=1$, $2, \ldots$, as $\mu E \rightarrow 0$. Hence, again by the Lemma, $\left\{T_{n}(|x-r|)\right\}_{n=1}^{\infty}$ is weakly sequentially compact. It follows that there exists a subsequence $T_{n_{j}}(|x-r|)$ which converges weakly to some $g \in L_{1}(0,1)$. Now let $E$ be any measurable subset of $(0,1)$. We have on account of $(1)$ and the positivity of the operators

$$
\int(x-r) \cdot \chi_{E} \leftarrow \int T_{n_{j}}(x-r) \cdot \chi_{E} \leqslant \int T_{n_{j}}(|x-r|) \cdot \chi_{E} \rightarrow \int g \cdot \chi_{E} .
$$

Received by the editors December 24, 1975.

AMS (MOS) subject classifications (1970). Primary 41A35; Secondary 47B99. 
Thus $x-r \leqslant g$ a.e. Similarly $r-x \leqslant g$ a.e. Therefore $|x-r| \leqslant g$ a.e. On the other hand, it follows from the contractive nature of the operators that $\|g\| \leqslant \underline{\lim }\left\|T_{n_{j}}(|x-r|)\right\| \leqslant\|x-r\|$. The above imply that $g=|x-r|$ a.e., in other words

$$
T_{n_{j}}(|x-r|) \stackrel{w}{\longrightarrow}|x-r| .
$$

By Cantor's diagonal argument, there exists a subsequence, say $\left\{l_{j}\right\}$, of $\left\{n_{j}\right\}$ such that

$$
T_{l j}(|x-r|) \stackrel{w}{\longrightarrow}|x-r|
$$

for every rational $r$.

The linear span of the functions $1, x,\{|x-r|: r$ rational $\}$ is the set of polygonal lines with rational vertices; hence it is dense in $L_{1}(0,1)$. From (1) and (3) it follows, therefore, that

$$
T_{l_{j}} \stackrel{w}{\longrightarrow} f
$$

for every $f \in L_{1}(0,1)$.

Since from every subsequence of $\left\{T_{n}\right\}$ we can select a further subsequence satisfying (4), (2) must be true for the sequence $\left\{T_{n}\right\}$ itself.

The following result of Wulbert [2] is an immediate consequence of the Theorem.

Corollary. Let $\left\{T_{n}\right\}_{n=1}^{\infty}$ be a sequence of positive and contractive linear operators on $L_{1}(0,1)$ satisfying

$$
T_{n} 1 \stackrel{s}{\rightarrow} 1, \quad T_{n} x \stackrel{w}{\longrightarrow} x .
$$

Then $T_{n} f \stackrel{s}{\rightarrow}$ for every $f \in L_{1}(0,1)$.

Proof. Let $E$ be any measurable subset of $(0,1)$ and $\tilde{E}$ its complement. Since $\chi_{E}=1-\chi_{\tilde{E}}$, we have on $(0,1)$

$$
T_{n} \chi_{E}-\chi_{E}=\left(T_{n} 1-1\right) \cdot \chi_{E}-\left(T_{n} \chi_{\tilde{E}}\right) \cdot \chi_{E}+\left(T_{n} \chi_{E}\right) \cdot \chi_{\tilde{E}} .
$$

Hence

$$
\left\|T_{n} \chi_{E}-\chi_{E}\right\| \leqslant\left\|T_{n} 1-1\right\|+\int\left(T_{n} \chi_{\tilde{E}}\right) \cdot \chi_{E}+\int\left(T_{n} \chi_{E}\right) \cdot \chi_{\tilde{E}} .
$$

The first quantity on the right side tends to zero on account of (7), the second and third tend to zero by our Theorem. Hence $T_{n} \chi_{E} \stackrel{s}{\rightarrow} \chi_{E}$ for every measurable $E$. The conclusion now follows, since $\left\{\chi_{E}\right\}$ are dense in $L_{1}(0,1)$.

REMARKs. (i) The assumption $\left\|T_{n}\right\| \leqslant 1$ cannot be removed. For, if

$$
(T f)(x)= \begin{cases}\frac{3}{2} \int_{x-1 / 3}^{x+1 / 3} f, & \frac{1}{3}<x<\frac{2}{3}, \\ f(x), & \text { otherwise. }\end{cases}
$$


Then $T 1 \equiv 1, T x \equiv x$, but $T \chi_{E}=\frac{1}{2} \chi_{E}$ for $E=\left[\frac{1}{3}, \frac{2}{3}\right]$.

(ii) The conclusion $T_{n} f \stackrel{w}{\longrightarrow} f$ cannot be replaced by $T_{n} f \stackrel{s}{\rightarrow} f$. For, if

$$
\left(T_{n} f\right)(x)= \begin{cases}2 n \int_{k / n}^{(k+1) / n} f, & \frac{k}{n}<x<\frac{2 k+1}{2 n} ; 0 \leqslant k<n, \\ 0, & \text { otherwise, }\end{cases}
$$

then $T_{n} f \stackrel{w}{\longrightarrow} f$ for every $f \in L_{1}(0,1)$, but $\left\|T_{n} 1-1\right\|=1$. This example is due to J. B. Gamlen.

\section{REFERENCES}

1. N. Dunford and J. T. Schwartz, Linear operators. I: General theory, Pure and Appl. Math., vol. 7, Interscience, New York, 1958. MR 22 \#302.

2. D. E. Wulbert, Convergence of operators and Koroukin's theorem, J. Approximation Theory 1 (1968), 381-390. MR 38 \#3679.

Department of Mathematics, University of Alberta, Edmonton, Alberta, Canada 